\title{
Uso de Kahoot como elemento motivador en el proceso enseñanza-aprendizaje
}

\section{Use of Kahoot as a motivating element in the teaching- learning process}

\author{
Juan Rojas-Viteri \\ Universidad Central del Ecuador, Quito, Ecuador \\ jcviteri@uce.edu.ec \\ https://orcid.org/0000-0002-7466-7364 \\ Alex Álvarez-Zurita \\ Instituto Tecnológico Superior Sucre, Quito, Ecuador \\ amalvarez@uce.edu.ec \\ https://orcid.org/0000-0003-4535-4534 \\ Diego Bracero-Huertas \\ Universidad Central del Ecuador, Quito, Ecuador \\ dgbracero@uce.edu.ec \\ https://orcid.org/0000-0001-5000-864X
}

(Recibido: 11/12/2021; Aceptado: 15/01/2021; Versión final recibida: 20/01/2021)

Cita del artículo: Rojas-Viteri, J., Álvarez-Zurita, A. y Bracero-Huertas, D. (2021). Uso de Kahoot como elemento motivador en el proceso enseñanza-aprendizaje. Revista Cátedra, $4(1), 98-114$.

\section{Resumen}

El presente estudio surgió de la necesidad de generar en el alumnado investigado el deseo de aprender mientras se divierten. Las metodologías activas de aprendizaje y, especialmente, la gamificación en la actualidad ha sido muy utilizada; existe en la web un sinfín de herramientas digitales que cumplen con este propósito. El objetivo general de la investigación fue analizar el uso de Kahoot como elemento motivador en el proceso enseñanza-aprendizaje. La metodología utilizada en la estructuración de este trabajo fue empírica-analítica de corte descriptivo. Además, se emplearon durante todo el período lectivo 2019-2020 cuestionarios tipo quiz para reforzar los temas tratados en cada clase,

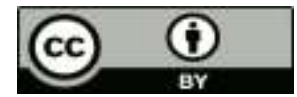


mismos que permitieron medir estadísticamente la preferencia del alumnado por el uso de Kahoot. La población estuvo determinada por treinta y cinco estudiantes que pertenecieron al segundo semestre de la Carrera de Pedagogía de las Ciencias Experimentales Informática de la Universidad Central del Ecuador (UCE). Los resultados revelaron que el alumnado investigado mostró estar motivado al momento de aprender, así como también al momento de ser evaluados, pues se sentían sin presión para responder. Finalmente, se demostró que Kahoot es una herramienta digital que motiva el proceso de enseñanza-aprendizaje incentivando al estudiantado a asistir a clases y participar de forma más activa en el aula.

\section{Palabras clave}

Enseñanza-aprendizaje, gamificación, Kahoot, motivación.

\section{Abstract}

The present study arose from the need to generate in the investigated students the desire to learn while having fun. Active learning methodologies and, especially, gamification have been widely used nowadays; there is an endless number of digital tools on the web that fulfill this purpose. The general objective of the research was to analyze the use of Kahoot as a motivating element in the teaching-learning process. The methodology used in structuring this work was empirical-analytical and descriptive. In addition, quiz-type questionnaires were used throughout the 2019-2020 school year to reinforce the topics covered in each class, which made it possible to statistically measure the students' preference for the use of Kahoot. The population was determined by thirty-five students who belonged to the second semester of the Pedagogy of Experimental Sciences and Computer Science course at the Central University of Ecuador (UCE). The results revealed that the students investigated showed to be motivated at the moment of learning, as well as at the moment of being evaluated, since they felt no pressure to respond. Finally, it was demonstrated that Kahoot is a digital tool that motivates the teaching-learning process, encouraging students to attend classes and participate more actively in the classroom.

\section{Keywords}

Teaching-learning, gamification, Kahoot, motivation.

\section{Introducción}

Actualmente nos encontramos conectados a Internet la mayoría de nuestro tiempo, inclusive, sin darnos cuenta se aumenta cada vez el uso de dispositivos digitales en las diferentes áreas de nuestra vida. Las nuevas formas de comunicarse permiten acceder a la información y al conocimiento, y para ello se hace uso de diferentes herramientas tecnológicas, aunque no se suela sacar todo el provecho que se debería, principalmente en el campo educativo.

El sector educativo es uno de los más afectados y al no seguir el ritmo de los nuevos avances en las tecnologías de la información y comunicación, ésta se ve afectada en el proceso de enseñanza-aprendizaje. Las dificultades que tienen los maestros para adaptarse a la utilización de estas herramientas tecnológicas es una realidad. Al respecto, Fonoll et al. (2011) indica que:

para el profesor de aula las tecnologías digitales suponen ya de por si un cambio en su forma de trabajar. Cuando menos, les supone cambios metodológicos en su planteamiento de actuación 
en el aula. Esto significa que el profesor debe adaptar los contenidos educativos a las necesidades del alumno (pág. 36).

De esta manera Sánchez considera que el uso adecuado de las herramientas digitales, puede motivar al alumnado en su proceso de aprendizaje. Tal como lo demuestran varios estudios realizados a nivel mundial (Sánchez, 2015). Es importante prestar atención a la distracción en los estudiantes, tanto en clases como cuando realizan tareas, pues los discentes pueden redirigir su atención a otras actividades que les parezcan más interesantes. Al respecto, García-Varcárcel, Muñoz-Repiso (2008) indica que:

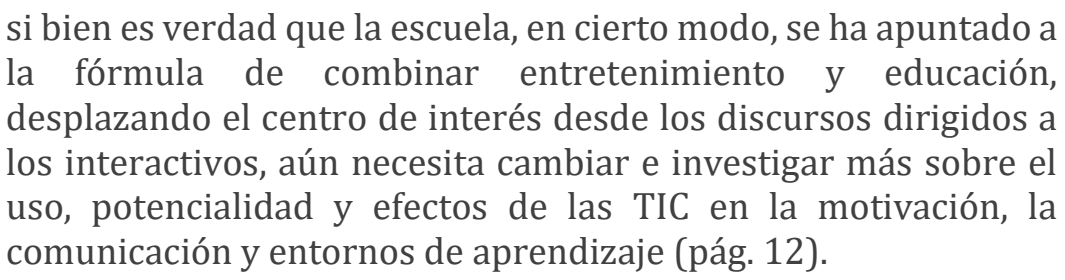

Así, se puede determinar que existen entornos educativos en línea que a través del juego buscan vencer el desinterés del alumnado por aprender. Es aquí donde la gamificación como técnica lúdica de aprendizaje se presenta como una alternativa para construir conocimientos a través del juego.

Uno de los componentes que intervienen en esta investigación es precisamente la evaluación sobre el uso de la herramienta Kahoot, considerada como una metodología lúdica, efectiva para los estudiantes. Al respecto, Teixes (2015) menciona que "han crecido entre videojuegos y están acostumbrados a feedback y recompensas inmediatos y constantes" (pág. 34). Estas técnicas que se utilizan en algunas apps de educación como, por ejemplo, Duolingo que utiliza recompensas y logros para enseñar idiomas. Por otra parte, Kahoot nos permite implementar la gamificación en todo tipo de asignaturas sin enfocarse en una en concreto.

Si bien la gamificación aumenta la motivación intrínseca del estudiante ya sea por el sentido de competencia al realizar un juego o por el ambiente de compañerismo, existen algunos factores que se deben tomar en cuenta para una correcta implementación de la gamificación en el aula. Gràcia, Sanlorien y Segués (2017) dice que, si el "reto es muy alto y la percepción subjetiva es de poca autoeficiencia, se crea ansiedad y, por el contrario, si la tarea es muy fácil y la idea de autoeficacia o sus capacidades son altas, entonces el individuo se aburre" (pág. 70). Si se plantea este concepto al uso de Kahoot en las clases significa que las preguntas que proporciona esta herramienta deben estar acorde al nivel de conocimiento del alumno, de forma que se plantee un reto alcanzable que a su vez produzca conocimiento, habilidades y destrezas. Kahoot es un recurso digital de aprendizaje que puede ser usado con diferentes propósitos, por ejemplo: diagnosticar conocimientos sobre un tema, conocer aspectos notables de una unidad o comprobar lo que se aprendió, así como también, evaluar el grado de comprensión de una lectura o debatir sobre un tema en concreto (Pintor et al., 2015).

En cuanto a la estructura del artículo, en la sección 2 se presenta los conceptos relacionados con la investigación. En la sección 3 se detalla los trabajos relacionados con este estudio. En la sección 4 se indica la metodología utilizada para la investigación. En la sección 5 se muestra los resultados del uso de la herramienta Kahoot. En la sección 6 se escribe las discusiones. Finalmente, en la sección 7 se establece las conclusiones. 


\section{Conceptos relacionados}

\subsection{Kahoot}

En la actualidad existen herramientas que facilitan todo tipo de tareas, en el caso de la educación se tiene facilidades para conseguir información, comunicarse o incluso realizar trabajos académicos. En el proceso enseñanza-aprendizaje se dispone de herramientas para tomar exámenes, enviar tareas y calificarlas. Kahoot como herramienta lúdica permite implementar una metodología de gamificación que da la posibilidad de realizar cuestionarios de forma dinámica. Gallegos (2015) argumenta que kahoot es:

una de las herramientas digitales gratuitas más divertidas, enganchadoras e innovadoras que un docente puede utilizar para aumentar el clima creativo de su aula de clase. Generalmente un clima creativo se caracteriza por el buen humor, la risa, la ausencia del temor al qué dirán, un ambiente de trabajo en equipo y una atmósfera de camaradería; esta mezcla de elementos hace que la clase sea más placentera, menos aburrida y mucho más motivante (pág. 48).

Kahoot, fue creado por el profesor Alf Inge Wang, quién pensó en un ambiente educativo cómodo y divertido. Esta herramienta basada en el juego es más que un simple test online y tiene ciertas características que invitan al estudiantado a participar utilizando cualquier dispositivo móvil, de manera accesible y fácil de utilizar, tanto para el docente como para los alumnos. Al respecto, Gallegos (2015) expresa:

Básicamente el docente crea las preguntas sobre cualquier tema o en cualquier idioma, y los estudiantes responden las preguntas en tiempo real desde cualquier dispositivo, ya sean estos celular, tableta o computadora. El estudiante que más rápido responde va ganando puntos, y créanme cuando les digo que cada vez que pruebo esta herramienta, ya sea con niños o adultos, el aprendizaje se activa, la gente se despierta y se aumenta la predisposición por aprender (pág. 48).

Con base en la cita anterior se puede observar que kahoot puede ser muy intuitivo en su forma de trabajar y tener las facilidades de acceso en diferentes dispositivos. Es evidente el aumento de motivación de los estudiantes que reciben sus clases utilizando esta herramienta, así expresan los docentes que lo han usado la herramienta; no importa la edad o tipo de asignatura.

\subsection{Gamificación}

Existen muchos conceptos de gamificación, pero por fines prácticos es recomendable utilizar la definición de Teixes (2015) quien considera que "la gamificación es la aplicación de recursos propios de los juegos (diseño, dinámica, elementos, etc.) en contextos no lúdicos, con el fin de modificar los comportamientos de los individuos, actuando sobre su motivación, para consecución de objetivos concretos" (pág. 18). Con esta apreciación se evidencia que la utilidad principal de la gamificación es el cambiar o modificar un comportamiento, pero esto se debe lograr de una manera más amigable y con la colaboración voluntaria de las personas.

Para complementar esta definición y entender de una forma más fácil, a criterio personal se puede definir a la gamificación como un juego. El juego provoca alegría y diversión. Y al 
combinar estas características de felicidad con la educación, se podría solucionar el problema de transformar una tarea que puede ser desmotivante o aburrida en una actividad altamente motivada y divertida. Ordás (2018) dice:

La gamificación se basa en una idea simple: a todos nos gusta jugar. A partir de ahí, introduce elementos de los juegos en entornos cotidianos, como las organizaciones, el marketing, la salud o la educación, con el objetivo de crear un nuevo ambiente que motive a las personas de esos entornos a participar en sus propuestas (pág.17).

Así, la estrategia de implementar elementos de los juegos se utiliza en diferentes áreas del conocimiento que tienen como objetivo motivar a las personas a utilizar cierto producto, a participar en una campaña o como en este caso a aprender. En educación se innova muy poco a diferencia de otras áreas como la tecnología y es esta misma tecnología la que proporciona la facilidad de crear nuevas estrategias para el aprendizaje.

\subsubsection{La gamificación como motivación intrínseca}

Una particularidad que se logra en los individuos a causa de los juegos es la generación de motivación para conseguir objetivos. Para Teixes (2015) "la motivación intrínseca es la tendencia inherente a buscar la novedad y el desafío, a extender y ejercitar las propias capacidades, a explorar y aprender" (pág.22). Este comportamiento del ser humano es aprovechado por la gamificación y sus elementos generando la motivación de forma natural.

\subsubsection{Características propias de la gamificación}

Gamificación es la aplicación de principios y elementos propios del juego en un ambiente de aprendizaje con el propósito de influir en el comportamiento, incrementar la motivación y favorecer la participación de los estudiantes. La gamificación es recurrir al uso de elementos que forman parte de la estructura del juego, es aplicar esta estrategia metodológica en una herramienta de apoyo docente que logre despertar motivación en los estudiantes con el fin que sus procesos de aprendizaje sean significativos y exitosos.

El feedback se trata de la manera de informar a los jugadores que tan lejos o cerca están de alcanzar el objetivo del juego, puede ser en forma de puntuación o directamente informando el ganador.

Participación voluntaria, los jugadores deben estar de acuerdo tanto con el objetivo planteado como con las normas y el feedback, de esta manera el jugador tiene una experiencia agradable (Teixes 2015, pág.27).

\subsection{Motivación en el aprendizaje}

En el proceso de aprendizaje se toman en cuenta diversos factores para que este se lleve a cabo de la mejor manera. Al respecto, Gallardo-Vásquez y Camacho-Herrera (2008) menciona que el aprendizaje es el "cambio en la conducta, debido a la experiencia que no puede explicarse por la maduración o por tendencias de respuesta innata" (pág. 23). Una de las mejores formas de alcanzar este cambio de conducta en los individuos es motivarlos en su aprendizaje.

La motivación es un factor muy importante en el proceso de aprendizaje, un estudiante motivado tendrá un mejor desempeño en sus destrezas y su desarrollo. Gallardo-Vásquez y Camacho-Herrera (2008) dice "el término motivación forma parte de nuestro lenguaje 
cotidiano y generalmente lo empleamos para referirnos a los 'motivos' o 'razones' que explican nuestro comportamiento o el comportamiento de los demás" (pág. 9). El deseo de aprender es algo que se puede lograr utilizando diferentes estrategias que generen motivación en el estudiante. La gamificación está centrada en el alumno porque puede ajustarse a las necesidades de cada individuo y motivarles a hacerse cargo de su propio aprendizaje.

\subsubsection{Rasgos que definen a una actividad motivada}

Para Kim reconocer los diferentes intereses y motivaciones de los participantes ayuda a desarrollar un ambiente atractivo para todos los estudiantes. Así pueden incorporarse elementos de juego que propicien en mayor medida el involucramiento y el consecuente desenvolvimiento de todos los participantes en la actividad (Kim, 2015).

Dirigida a metas u objetivos: los valores e intereses personales
pueden satisfacerse cumpliendo ciertos propósitos a esto apunta
la motivación de una actividad.

Autorregulación: implica la evaluación de los resultados obtenidos además de procesos que posibiliten la realización de la actividad (Gallardo-Vásquez, Camacho-Herrera, 2008, pág.10).

\subsubsection{Factores que influyen en la motivación}

Para Kapp la motivación puede ser intrínseca o extrínseca. La motivación intrínseca ocurre cuando una recompensa surge de la realización de una actividad. La motivación extrínseca alimenta el comportamiento realizado específicamente para obtener una recompensa externa. La motivación intrínseca suele tener mayor valor educativo. Los juegos bien diseñados presentan ambos tipos de motivación (Kapp, 2015).

Factores sociales: la imitación y competencia ya sea solo o entre grupos son factores que generan gran motivación. Factores Didácticos: son premios, castigos vienen de factores externos como son los resultados de evaluaciones, el contexto es parte de estos factores (Gallardo-Vásquez, Camacho-Herrera, 2008, pág.18).

\section{Trabajos relacionados}

En el año 2019, en Ecuador se realizó una investigación cuasi - experimental, donde se trabajó con un grupo de estudiantes de décimo año EGB, mediante la implementación de la metodología gamificación. En los resultados se comprobó que incide en el proceso de enseñanza aprendizaje de ecuaciones cuadráticas. Los autores concluyen que se notó aumento de interés del grupo durante el desarrollo de las clases (Sánchez, 2015).

También encontramos que, en el año 2019, en Ecuador se realizó otra investigación mediante la implementación de un software educativo, para el aprendizaje de matemática básica elemental en la resolución de operaciones mediante la gamificación. Los resultados fueron que los discentes se sintieron atraídos por superar retos y niveles, además de mejorar la cooperación y el compañerismo. Los autores concluyen que permite al docente reforzar lo aprendido en clase Gutiérrez-Constante y Herrera-Oña (2019).

Otra investigación cuasi-experimental que encontramos en Ecuador se realizó en el año 2017 y contó con la participación de cincuenta estudiantes de nivel pre-intermedio 
(mediante un diagnóstico, para posteriormente implementar la gamificación). Los resultados comprobaron la efectividad de la gamificación como estrategia de aprendizaje en la producción escrita del idioma inglés. Los autores concluyen que se evidenció su uso como una estrategia motivadora que promueve el aprendizaje de una manera dinámica y alegre Díaz-Villarruel y Cerda-Solís (2018).

Para el 2018, se realizó una investigación en Ecuador que mostró a la gamificación como una estrategia de aprendizaje para el apoyo de matemáticas en el tema de polinomios. Como resultado de este estudio se comprobó un aumento en el rendimiento académico del alumnado y se demostró que la gamificación aportó como estrategia de aprendizaje en Matemáticas, los autores concluyeron que el desconocimiento de estas herramientas hace que sean desaprovechadas Pilamunga-Poveda y Quizhpi-Lupercio (2018).

Para el año 2018, en Ecuador se realizó una investigación sobre la gamificación y el razonamiento verbal de los estudiantes de bachillerato de la Unidad Educativa Santo Domingo de Guzmán, en la ciudad de Ambato. Esta investigación se realizó con un enfoque cuantitativo. Los resultados obtenidos fueron que la gamificación ayuda en el desarrollo del razonamiento verbal de los estudiantes de tercero de bachillerato. Los autores concluyen que las clases de razonamiento verbal se desarrollan de forma tradicional con poco uso de tecnologías Páez-Quinde y Crespo-Jara (2018).

En el año 2017, en Ecuador se realizó una investigación sobre patrones en gamificación y juegos serios, aplicados a la educación; esta se llevó a cabo en la Carrera de Psicología Industrial de la Facultad de Ciencias Humanas y de la Educación de la Universidad Técnica de Ambato. Como resultado de ese estudio se comprobó que la gamificación y los juegos serios, implementados al proceso de aprendizaje, favorecen al desarrollo mental y las habilidades cognitivas. Los autores concluyen que el alumnado aprendería de mejor manera si utilizara los juegos serios en el proceso de aprendizaje Gómez-Alvarado y Loján-Carrión (2017).

En el 2018, en Colombia se realizó una investigación en la Pontificia Universidad Javeriana en la que se observó a dos grupos universitarios de distintas carreras y semestres. Utilizando la metodología de gamificación para aumentar la motivación de los estudiantes. Como resultado de esta investigación se evidenció de un aumento en la satisfacción, motivación y entusiasmo de los estudiantes, esto se vio reflejado en sus calificaciones. Los autores concluyen que sin darse cuenta los estudiantes estaban aprendiendo significativamente (Ardila, 2018).

En el 2018, en Perú se realizó una investigación en la Universidad César Vallejo. Se implementó el programa de gamificación autoconstructiva a estudiantes de primer grado de primaria del Callao. En este estudio se usó un enfoque cuantitativo. Los resultados indicaron una diferencia significativa a nivel estadístico de mejora en las capacidades de representación simbólica y representación algorítmica de los estudiantes. Los autores concluyen que la gamificación auto -constructiva fue decisiva para aceptar sus hipótesis (Chávez, 2018).

\section{Metodología}

El presente estudio abordó el uso de una herramienta digital, basada en el juego, aunque se ha profundizado en la gamificación como técnica lúdica para lograr aprendizajes. Se pretendió verificar la eficacia del uso de Kahoot en el salón de clases, para esto, se usó una metodología empírica- analítica, de corte descriptivo, midiendo a través de las frecuencias 
de uso, la eficacia de esta aplicación educativa. El objetivo que motivó este estudio fue analizar el uso de Kahoot como elemento motivador en el proceso enseñanza-aprendizaje.

Así, se empleó durante todo el semestre cuestionarios tipo quiz referentes al tema tratado al finalizar cada clase. El grupo investigado estuvo constituido por 35 estudiantes del 2do semestre de la asignatura tecnología educativa, de la Carrera de Pedagogía de las Ciencias Experimentales Informática de la UCE.

El procedimiento consistió, en que el docente de la materia diseñaba los cuestionarios en Kahoot, con base en los contenidos a tratar en cada clase y una vez finalizada esta se los aplicaba al estudiantado para evaluar su aprendizaje en cada sesión. Por su parte, los discentes usaban sus dispositivos móviles para contestar las preguntas, pues esta herramienta digital es muy flexible. Según Fernández et al. la intuitiva interfaz gráfica del aplicativo favorece su uso, el alumno la percibe como un juego y no como un sistema de evaluación (Fernández et al., 2016, p. 18). Fue evidente la motivación del alumnado cada vez que participaba, contestaban las preguntas a través de los dispositivos tecnológicos y esto incrementaba la colaboración de todo el grupo en la actividad, sin que notaran que aprendían jugando.

En este grupo se aplicó un cuestionario conformado por 36 preguntas, con un baremo de 5 posibilidades en la escala de Likert, para recoger las experiencias vividas con el uso de Kahoot. El instrumento fue validado por 3 docentes -expertos de la carrera en cuestión, mismos que contribuyeron con sus observaciones a la mejora del mismo.

Para medir el grado de consistencia interna, se aplicó el coeficiente de fiabilidad alfa de Cronbach cuyo valor resultante fue de ,870 equivalente a bueno. Este análisis trata de determinar el grado en el que están relacionados recíprocamente los reactivos o ítems (Brown, 1980) y lo presentamos a continuación:

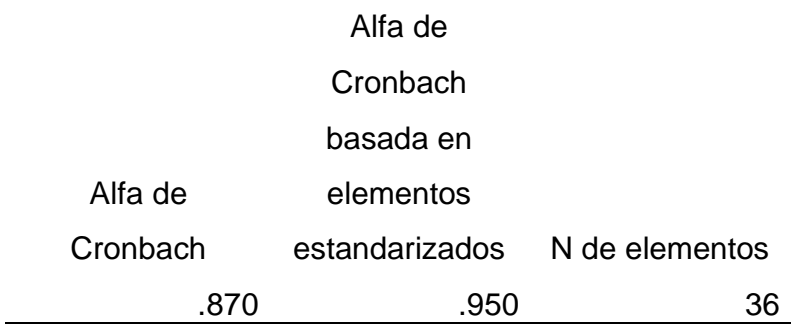

Cuadro 1. Alfa de Cronbach

\section{Resultados}

Atendiendo a la responsabilidad de cumplir con un proceso científico, el estudio se lo realizó durante todo el semestre de clases, para que los resultados tuvieran un peso significativo, una vez se aplicó el instrumento para recoger y analizar la información. Es importante destacar que, en las evaluaciones de mitad y fin de semestre, se incluyeron algunas de las preguntas utilizadas en Kahoot, para corroborar la efectividad de esta aplicación, siendo los resultados bastante esclarecedores al momento de contestar las preguntas de manera asertiva por parte del estudiantado de dicha asignatura. A continuación, presentamos algunos de los resultados más importantes de este estudio: 


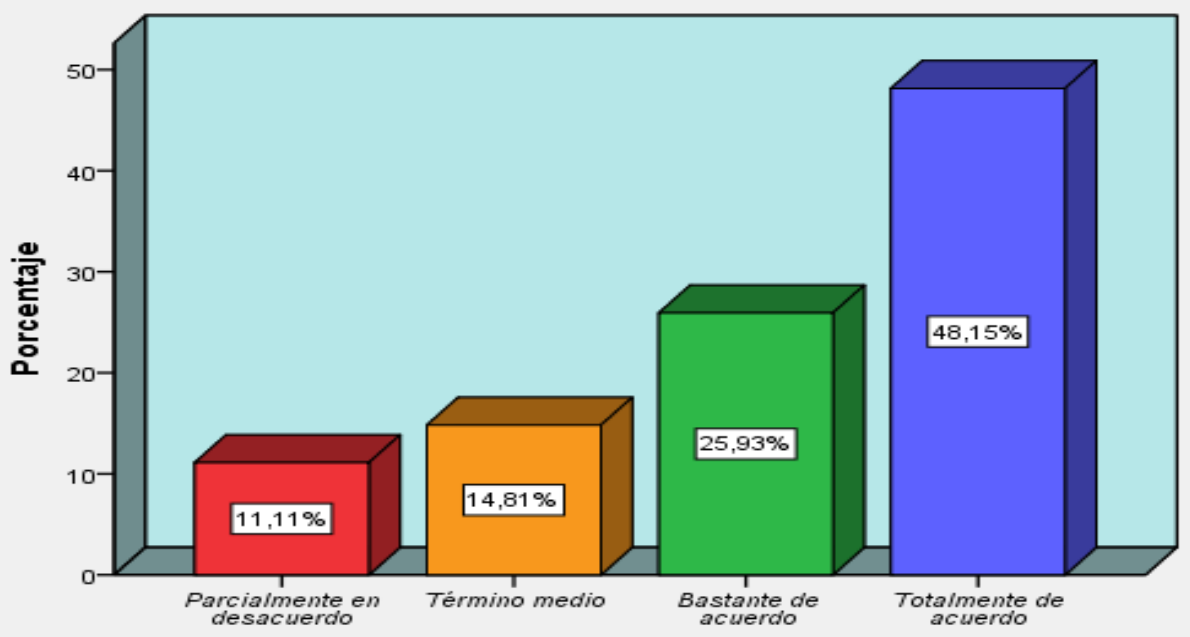

Figura 1. Experiencia del estudiantado al usar Kahoot sobre los temas tratados en clase

Desde hace muchas décadas atrás, los docentes e investigadores han ido incorporando distintos recursos tecnológicos que permitan mejorar el aprendizaje en los estudiantes. Es así que en los salones de clase se ha pasado de usar la radio y la tv a las computadoras y los dispositivos móviles. Así también, los retroproyectores que fueron muy usados en el pasado y ahora empleamos diapositivas. Castells (2001) afirma: "Internet permite trabajar desde cualquier sitio (oficina móvil, oficina portátil, conexión ubicua...), pero no se está desarrollando el trabajo en casa." (p. 4). A la luz de los resultados obtenidos, podemos expresar que un $11.11 \%$ del alumnado manifestó estar parcialmente de acuerdo, un $14.81 \%$ expresó estar medianamente de acuerdo, un 25,93\% declaró estar bastante de acuerdo y un 48.15\% indicó encontrarse totalmente de acuerdo en que Kahoot, le permitió hacer un repaso de los temas tratados en clase. Como se puede evidenciar los resultados del análisis de los datos de los discentes, muestran una actitud positiva hacia el uso de esta herramienta digital en el proceso de enseñanza-aprendizaje de los investigados.

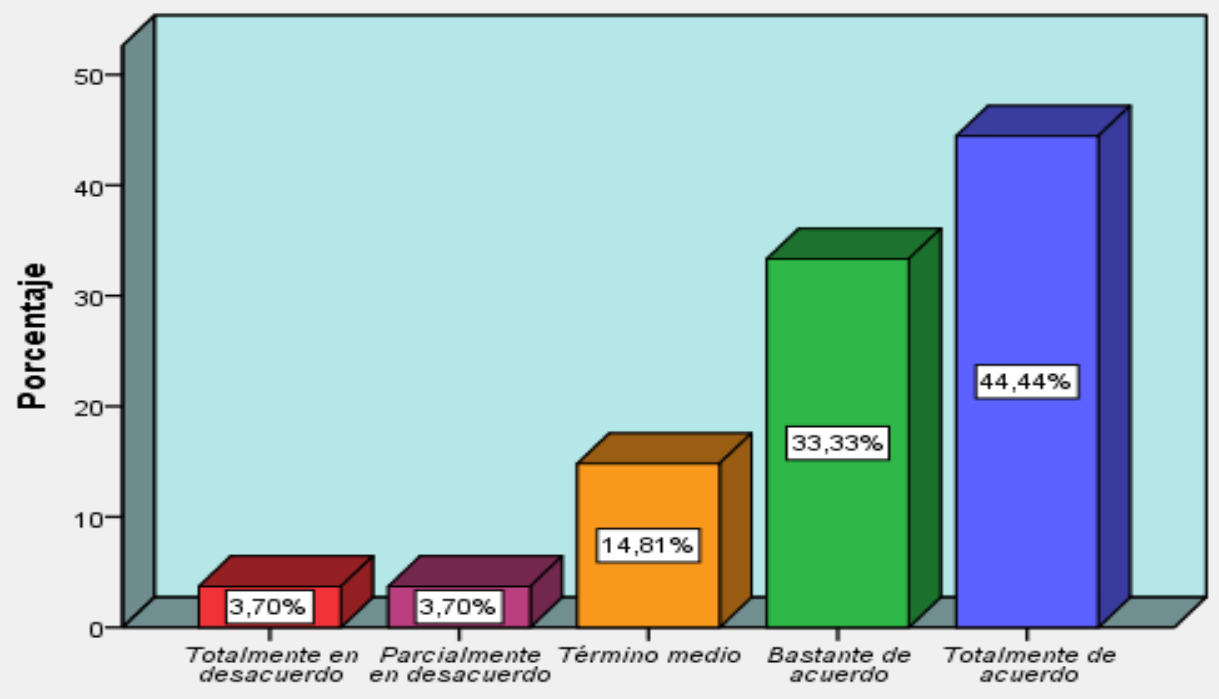

Figura 2. Preferencia del estudiantado al contestar preguntas a través de PC, celular o papel 
Las tecnologías de la información y la comunicación (TIC) son un conjunto de elementos y técnicas que permiten manipular la información, convertirla, almacenarla, administrarla, transmitirla y encontrarla, por medio del uso de computadoras y software a través de redes informáticas (Windschitl y Sahl, 2002). En este sentido podemos evidenciar que al momento de consultarles a los estudiantes sobre su preferencia de contestar preguntas a través del PC, celular o papel 3.70\% expresa estarlo totalmente en desacuerdo, el 3.70\% manifiesta estar parcialmente en desacuerdo, el $14.81 \%$ indica estar medianamente de acuerdo, el $33.33 \%$ manifiesta estar bastante de acuerdo, y el $44.44 \%$ de los discentes investigados expresan estar totalmente de acuerdo en la misma preferencia. Como podemos evidenciar los resultados son muy contundentes al expresar un favoritismo por el uso de las TIC, al momento de realizar sus evaluaciones, ya que prácticamente han convivido con ellas desde que son niños.

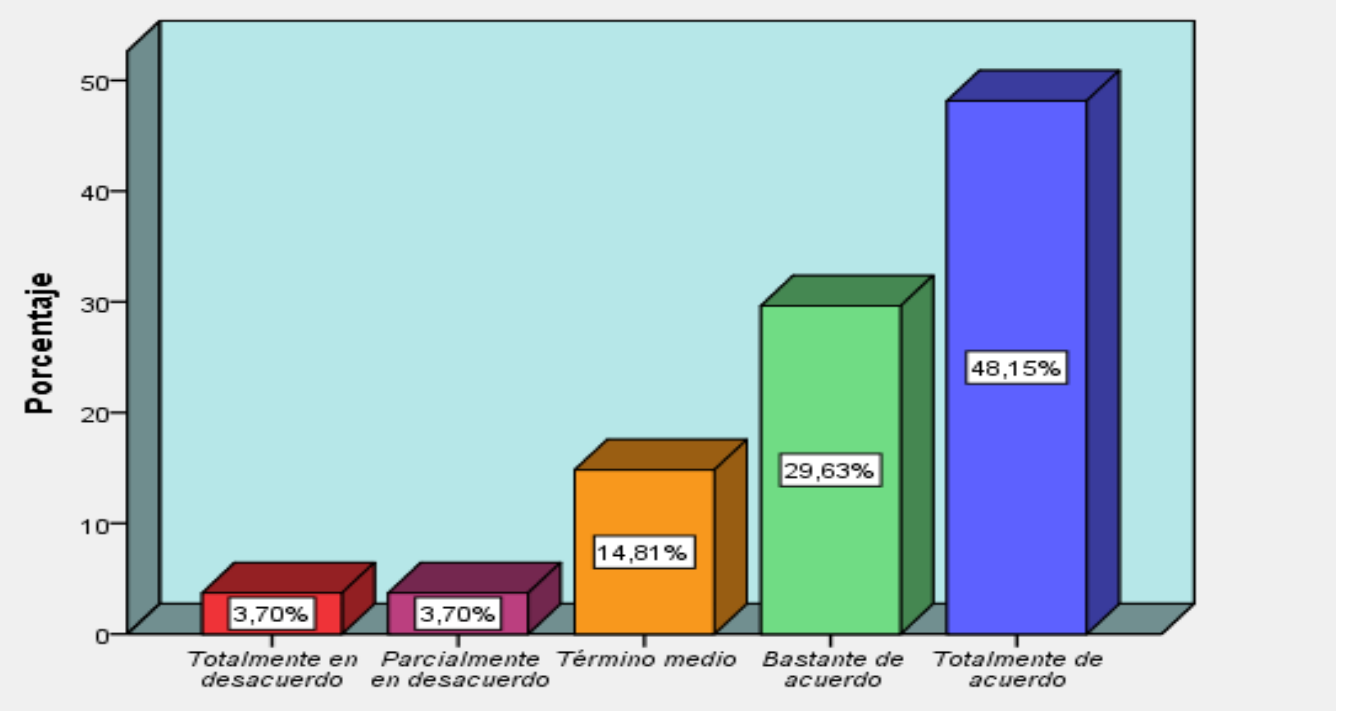

Figura 3. Motivación del estudiantado para participar en retos de preguntas en Kahoot

Según Wang existen algunas técnicas de formación y motivación nuevas, entre otras, las basadas en los juegos en el aula (game-based learning), usadas para realizar cuestionarios y diversión online en el aula de clases, favoreciendo la motivación mediante la competición entre los alumnos (Wang y Lieberoth 2015). Esta competencia entre el alumnado, se ve opacada por el trabajo en equipo, que en la mayoría de los casos generan estas herramientas. La motivación, es un aspecto muy importante en el proceso de enseñanzaaprendizaje, siendo esta, una característica de Kahoot. Así tenemos que un 3.70 \% de los investigados expresan estar totalmente en desacuerdo, el 3.70 manifiesta estar parcialmente en desacuerdo, el $14.81 \%$ indica estar medianamente de acuerdo, el $29.63 \%$, sostiene estar bastante de acuerdo y el $48.15 \%$ indica estar totalmente de acuerdo en sentirse motivados a estudiar y así participar en las preguntas a través de Kahoot. Aquí también los resultados son bastante concluyentes al indicar, donde se concentra la preferencia del alumnado. 


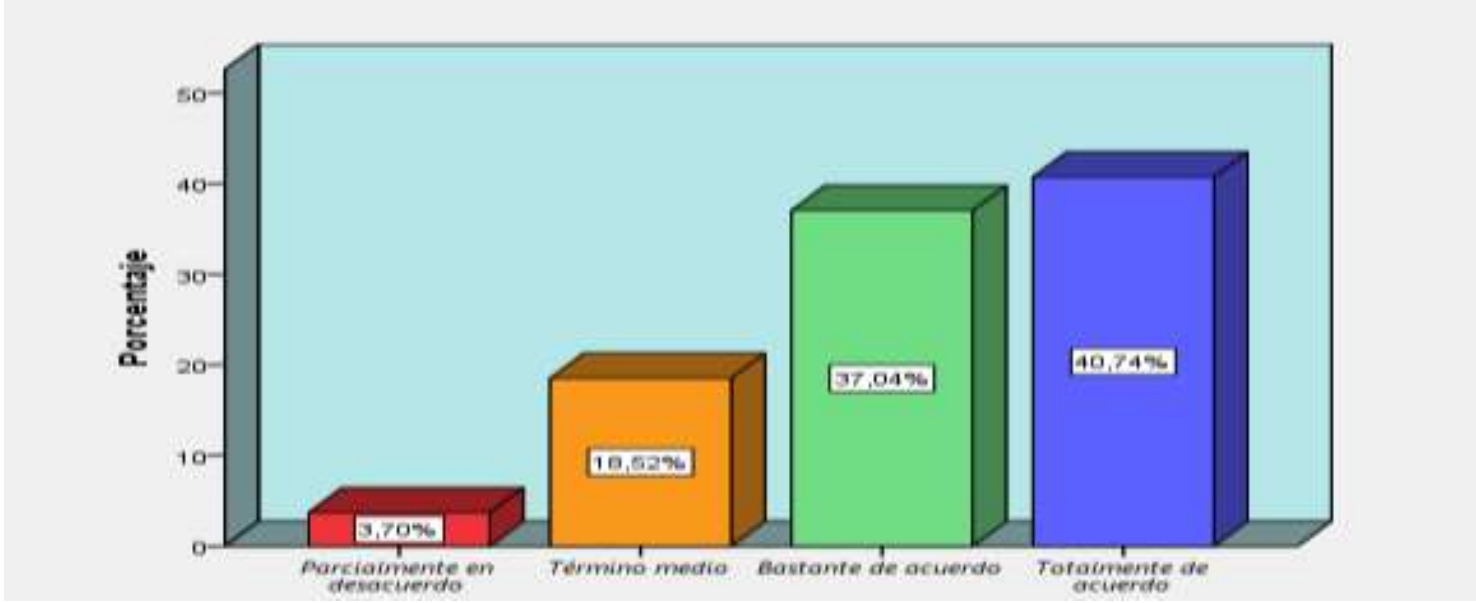

Figura 4. Importancia para el aprendizaje en el estudiantado al usar Kahoot

Entre otras, una de las primordiales ventajas de esta nueva manera de aprender es la colaboración activa de todo el alumnado. Los estudiantes menos participativos e introvertidos, que aportan, pero no sobresalen del resto del salón, reaparecen en el aula al lograr puestos destacados en el score. Esta metodología de aprendizaje, permite acortar la "distancia" con los alumnos distantes, una característica de los enfoques de enseñanza centrados en el estudiante (Salinas, 2004). La apreciación del alumnado respecto a la importancia del uso de Kahoot es evidente. Y de los resultados obtenidos podemos ver que un $3.70 \%$ de los investigados expresan estar parcialmente en desacuerdo, el $18.52 \%$ manifiestan estar medianamente de acuerdo, el 37.04\% indican estar bastante de acuerdo y el $40.74 \%$ expresan estar totalmente de acuerdo en la importancia de usar Kahoot para su aprendizaje. Los resultados una vez más indican la preferencia de los alumnos por el uso de esta herramienta digital en su aprendizaje, lo que no es una novedad, ya que, al ser un recurso interactivo, resulta muy llamativo al estudiantado.

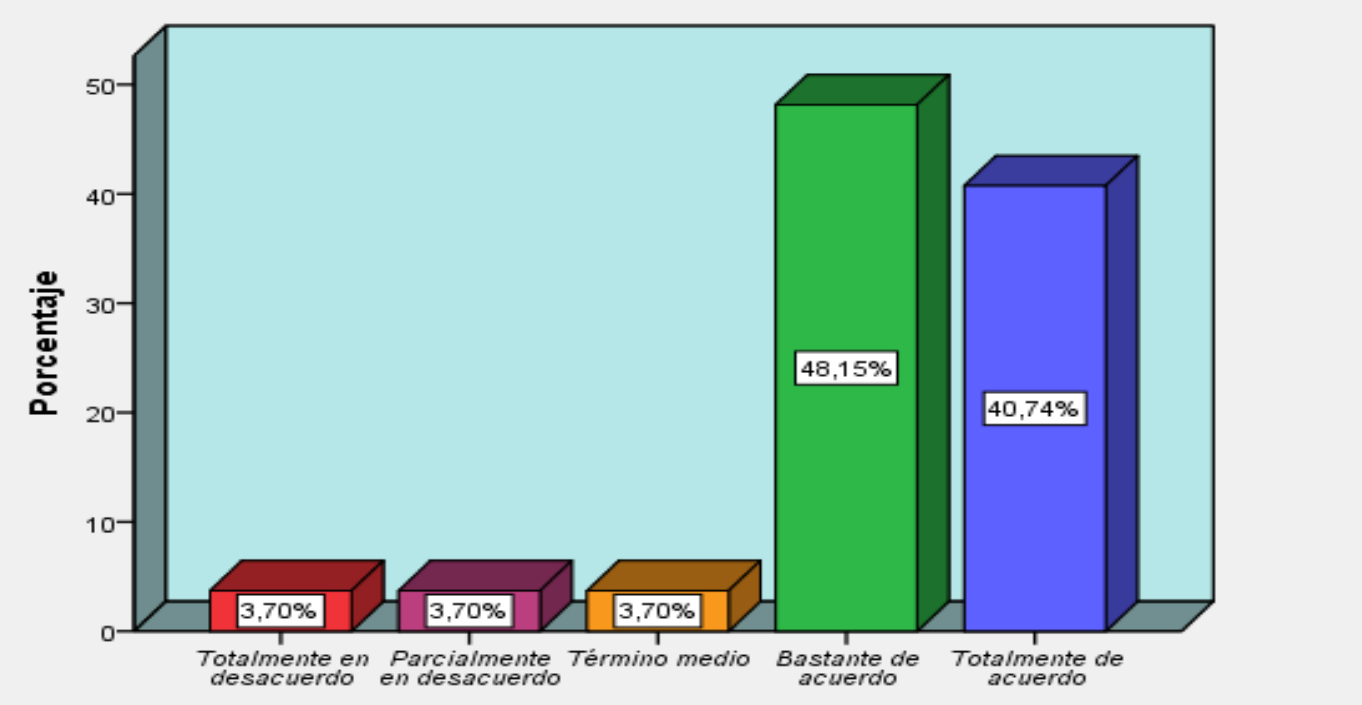

Figura 5. Desarrollo de aprendizaje colaborativo al usar Kahoot

Scardamalia y Bereiter (1994) afirman que "los estudiantes necesitan aprender profundamente y aprender cómo aprender, cómo formular preguntas y seguir líneas de 
investigación, de tal forma que ellos puedan construir nuevo conocimiento a partir de lo que conocen. El conocimiento propio que es discutido en grupo, motiva la construcción de nuevo conocimiento" (p. 266). Kahoot, provoca un aprendizaje colaborativo cuando los estudiantes discuten los resultados de las preguntas con el profesor, por este motivo es importante que el docente planifique de manera adecuada, no solo el cuestionario, sino cómo la va usar con el estudiantado. Como se puede observar y a la luz de los resultados obtenidos el $40.74 \%$ de los investigados enuncian estar totalmente de acuerdo en que Kahoot motiva el aprendizaje colaborativo. Así también, el 48.15\% de los investigados dan a conocer que están bastante de acuerdo con que Kahoot motiva el aprendizaje colaborativo. Por el contrario, un $11.10 \%$ de los consultados, manifiestan estar medianamente, parcialmente en desacuerdo y totalmente en desacuerdo con que el recurso TIC en cuestión, motiva el aprendizaje colaborativo.

\section{Discusión}

El software Kahoot es una importante y potente herramienta digital, que a través del aprendizaje basado en el juego logra que el estudiantado aprenda temas nuevos o repase otros ya aprendidos. Aquí yace la importancia del uso de estos recursos TIC en los salones de clase, dada la flexibilidad que presentan. Al analizar el uso de Kahoot como elemento motivador en el proceso enseñanza-aprendizaje y como se pudo observar en el apartado de los resultados, este software produce motivación al usarlo en el salón de clase. Así Rodríguez (2015) manifiesta: "este hecho transforma a este tipo de experiencias en un aliciente para su asistencia a clase (p. 12).

También, se puede evidenciar que los resultados presentados en las respuestas del cuestionario con respecto a la preferencia que el alumnado tiene al contestar las preguntas de evaluaciones a través del celular, en vez de utilizar papel, son mayoritariamente positivos para este estudio, pues exponen una realidad del estudiantado en los salones de clase. Y esto concuerda con una serie de estudios realizados por la Organización de las Naciones Unidas para la Educación, la Ciencia y la Cultura UNESCO (2013) quiénes han expuesto que "los dispositivos móviles pueden ayudar a los instructores a emplear con mayor eficacia el tiempo de clase" (p. 14).

Respecto a la motivación que les produce participar en los retos de Kahoot, los discentes expresaron mayoritariamente sentirse estimulados, cuando lo usan y el desafío que representa concursar y jugar entre compañeros, pero a la vez aprendiendo los contenidos tratados en clase. Varias investigaciones se han desarrollado en torno al juego y su eficacia en el proceso de enseñanza-aprendizaje. Al respecto, Guimaraes (2015), nos expone que "aprender jugando, resolviendo: diseñando experiencias positivas de aprendizaje" (p. 3). La mayoría de los estudiantes una vez a que han trabajado con Kahoot consideran que esta herramienta digital les permite aprender de manera dinámica gracias al juego. La gamificación ha sido usada en varias instituciones de educación básica, media y superior, en España y Europa en general, con muy buenos resultados. Rodríguez indica que Alf Inge Wang, -creador de Kahoot- aseveró en el estudio que ejecutó en Norwegian University of Science and Technology (NTNU) que los alumnos que manejaron este software, asimilaron un 22\% más que los estudiantes que manipularon juegos diferentes (Rodríguez, 2017).

Así tenemos que el estudiantado investigado, se manifestó mayoritariamente a favor que el uso de Kahoot, desarrolla aprendizaje colaborativo. Y esto es claro, ya que el uso de la gamificación como parte de la metodología docente ha generado nuevas oportunidades a educadores y estudiantes, permitiéndoles intercambiar experiencias y conocimientos y al mismo tiempo divertirse. 


\section{Conclusiones}

Una vez terminado el estudio y con los datos obtenidos se podemos aseverar, que Kahoot sí es un elemento motivador en el proceso de enseñanza-aprendizaje. Aunque sugerimos seguir investigando otras dimensiones involucradas para fortalecer los resultados de esta investigación. Recalcamos que, aunque los datos presentados en esta investigación a través del estudio de campo son precisos y claros, Kahoot se convirtió en una estrategia metodológica importante para el docente, pues incentivó al estudiantado a no faltar a clases. Además, de lograr una participación más activa en el aula.

Entre otras bondades de usar este software, es que posee una variedad de funciones importantes dentro de él, ya que dispone de múltiples estrategias para conseguir el objetivo que los estudiantes aprendan a través del juego. Es importante señalar que el profesorado que se incline por usar este programa informático debe ser competente, digitalmente hablando, dadas las distintas actividades a realizar.

Así también es importante señalar que el docente debe mantener un nivel de liderazgo significativo para llevar un adecuado orden en el salón de clase; en algunas ocasiones la algarabía de los estudiantes provocada por la participación y la competencia puede desencadenar desorganización e indisciplina dentro del aula y se requiere que el profesor logre reorganizar y redireccionar la atención de los estudiantes nuevamente a su aprendizaje. 


\section{Agradecimientos}

Un agradecimiento especial a la MSc. Simbaña-Gallardo Verónica por su guía en este trabajo investigativo. Además, extendemos nuestros agradecimientos a las autoridades y estudiantes de la Carrera de Ciencias Experimentales de la Informática por permitir que este estudio se lleve a cabo.

\section{Bibliografía}

Ardila, C. (2018). Crear, innovar y motivar en el aula gamificada. Trabajo de grado de Licenciatura en Pedagogía Infantil, Pontificia Universidad Javeriana, Bogotá. https://repository.javeriana.edu.co/bitstream/handle/10554/39771/Ivonne\%20A rdila $\% 20-\% 20$ tesis $\% 20$ gamificacio\%cc\%81n.pdf?sequence=4\&isAllowed=y

Castells, M. (2001). Internet y la sociedad en red. En Lección inaugural del programa de doctorado sobre la sociedad de la información y el conocimiento. Barcelona: UOC http://www.peremarques.net/si.htm

Díaz-Villarruel, J., Cerda-Solís, G. (2018). La gamificación como estrategia correctiva para la interferencia sintáctico-morfológica del español en la producción escrita del idioma inglés de los estudiantes de nivel pre-intermedio del Programa de Suficiencia en la Universidad de las Fuerzas Armadas ESPE extensión Latacunga durante el período octubre 2016 - marzo 2017. (Trabajo de titulación de Magíster en Lingüística y Didáctica de la Enseñanza de Idiomas Extranjeros). Universidad Central del Ecuador, Quito. pp.148 http://www.dspace.uce.edu.ec/handle/25000/16263

Chávez, Y. (2018). Programa de gamificación auto constructiva en el aprendizaje de adición y sustracción del primer grado de primaria del Callao. (Tesis de licenciatura en educación primaria. Universidad César Vallejo). Lima-Perú https://hdl.handle.net/20.500.12692/25042

Fernández, A., Olmos, J., Alegre, J., (2016). Valor pedagógico del repositorio común de conocimientos para cursos de dirección de empresas. Revista d'innovació Educativa, 16. https://www.redalyc.org/pdf/3495/349546195005.pdf

Fonoll-Salvador, J., Garcia-Fernández, J., Garcia-Villalobos, J., Guerra-Álvarez, A., Gutiérrez y Restrepo, E., Jaúdenes-Casaubón, C., Martínez-Normand, L., Romero-Zúnica, R. (2011). Accesibilidad, TIC y educación. Ministerio de Educación, Cultura y Deporte de España, Madrid.

\section{https://www.digitaliapublishing.com/a/14716/accesibilidad--tic-y-educacion}

García-Varcárcel, A., Muñoz-Repiso. (2008) Investigación y tecnologías de la información y comunicación al servicio de la innovación educativa. España: Ediciones Universidad de Salamanca. https://dialnet.unirioja.es/servlet/libro?codigo=352526

Gallardo-Vásquez, P., Camacho-Herrera, J. (2008) La motivación y el aprendizaje en educación, España: Wanceulen Editorial. https://books.google.com.ec/books?id=2t8ADAAAQBAJ\&lpg=PP1\&hl=es\&pg=PA3\# $\mathrm{v}=$ onepage $\& \mathrm{q} \& \mathrm{f}=$ false 
Gallegos, J. (2015). Kahoot, la mejor manera de aprender es jugando. Revista para el aula IDEA, 1(16), 48. https://issuu.com/usfq/docs/para el aula 16 diciembre 2015

Gràcia, M., Sanlorien P., Segués M. (2017). Motivación, afecto y relaciones en el aula inclusiva. Universitat Oberta de Catalunya, Barcelona.

Gutiérrez-Constante, G., Herrera-Oña, I. (2019). Diseño de un software educativo para el aprendizaje de matemática básica elemental en la resolución de operaciones mediante la gamificación en los estudiantes de la Academia Militar Saulo. (Trabajo de titulación de Licenciatura en Ciencias de la Educación, Mención Informática aplicada a la Educación. Universidad Central del Ecuador, Quito). http://www.dspace.uce.edu.ec/handle/25000/18454

Gómez-Alvarado, H., Loján-Carrión, M. (2017). Patrones en gamificación y juegos serios, aplicados a la educación. (Trabajo de Grado de Maestría en Informática Educativa. Universidad Técnica de Ambato, Ambato). http://repositorio.uta.edu.ec/jspui/handle/123456789/26793

Guimares, D. (2015). Kahoot: quizzes, debates e sondagens. En Carvalho, A. A. A. (2015). Apps para dispositivos móveis: manual para professores, formadores e bibliotecários (pp. 203-224). Disponible en: http://goo.gl/btf1G3

Kapp, K. (2012). The Gamification of learning and instruction. San Francisco: Pfeiffer.

Kim, B. (2015). Understanding Gamification. Obtenido de Library Technology Reports,

\section{1(2): https://journals.ala.org/ltr/issue/download/502/252}

Meneses-Rodríguez, W., Duque-Sánchez, P. (2017). Incidencia de la metodología gamificación en el proceso de enseñanza-aprendizaje de ecuaciones cuadráticas, en el décimo año EGB, de la Unidad Educativa Municipal Calderón, del Distrito Metropolitano de Quito, en el año lectivo 2018-2019. (Trabajo de titulación de Licenciatura en Ciencias de la Educación. Mención Matemática y Física). Universidad Central del Ecuador, Quito. pp.209 Recuperado de http://www.dspace.uce.edu.ec/handle/25000/18462

Ordás, A. (2018). Gamificación en bibliotecas: el juego como inspiración. España: Editorial UOC

Páez-Quinde, M., Crespo-Jara, F. (2018). Gamificación y el razonamiento verbal en los estudiantes de bachillerato. (Trabajo de grado de Maestría en Informática Educativa). Universidad Técnica de Ambato, Ambato. http://repositorio.uta.edu.ec/jspui/handle/123456789/28893

Pilamunga-Poveda, E., Quizhpi-Lupercio, L. (2018). La estrategia de gamificación y el proceso de aprendizaje. (Trabajo de Grado de Maestría en Informática Educativa. Universidad técnica de Ambato). Universidad Técnica de Ambato. http://repositorio.uta.edu.ec/ispui/handle/123456789/28903 
Pintor, E., Gargantilla, P., Herreros, B., López del Hierro, M. (2015). Kahoot en docencia: una alternativa práctica a los clickers. Disponible en: http://hdl.handle.net/11268/3603. Consultado el 02.01.2017.

Rodríguez, L. (2015). Role playing 2.0 en el grado de periodismo en Periodismo Digital y televisivo. Un reto profesional y de innovación docente en la universidad. Editorial: Dykinson.

https://www.researchgate.net/publication/331224183 Role Playing 20 su aplicac ion en el grado de periodismo

Rodríguez, L. (2017). Smartphones y aprendizaje: el uso de Kahoot en el aula universitaria. Revista Mediterránea de Comunicación/Mediterranean Journal of Communication, 8(1), 181-190. https://www.doi.org/10.14198/MEDCOM2017.8.1.13

Scardamalia, M., Bereiter, C. (1994). Computer Support for Knowledge-Building Communities, The Journal of the Learning Sciences, Vol. 3. No.3, pp. 265-283. http://etec.ctlt.ubc.ca/510wiki/images/5/5d/1466822.pdf

Salinas, J. (2004). Innovación docente y uso de las TIC en la enseñanza universitaria. RUSC. Universities and Knowledge Society Journal, 1(1),1-16. https://www.redalyc.org/articulo.oa?id=780/78011256001

Sánchez, F. (2015). Gamificación. Education in the Knowledge Society, vol. 16, núm. 2, 2015, pp. 13-15. Universidad de Salamanca. Salamanca, España. https://www.redalyc.org/articulo.oa?id=535554758002

Teixes, F. (2015). Gamificación: fundamentos y aplicaciones. Barcelona, España: Editorial UOC.

Organización de las Naciones Unidas para la Educación, la Ciencia y la Cultura. (2013). Aprendizaje Móvil. Recuperado de: https://es.unesco.org/themes/ticeducacion/aprendizaje-movil

Wang, A. y Lieberoth, A. (2015). The effect of points and audio on concentration, engagement, enjoyment, learning, motivation, and classroom dynamics using Kahoot! (Tesis Doctoral). Norwegian University of Science and Technology, Aarhus University, Interacting Minds Center (IMC) and Department of Education.

Windschitl, M. y Sahl, K. (2002). "Tracing teachers' use of technology in a laptop computer school: the interplay of teacher beliefs, social dynamics and institutional culture", American Educational Research Journal, vol. 39, núm. 1, pp. 165-205. http://www.scielo.org.mx/scielo.php?pid=S14056662013000200011\&script=sci ar $\underline{\text { ttext }}$ 


\section{Autores}

JUAN ROJAS-VITERI Licenciado en Educación Mención Informática en la Facultad de Filosofía, Letras de Ciencias de la Educación de la Universidad Central del Ecuador en 2002. Especialista en entornos virtuales de Aprendizaje (OEI). Magíster en Educación Superior en la Facultad de Filosofía, Letras de Ciencias de la Educación de la Universidad Central del Ecuador en 2012. Doctor en Investigación educativa por la Universidad de Alicante España.

Actualmente es profesor titular de la Facultad de Filosofía, Letras y Ciencias de la Educación de la Universidad Central Ecuador. Docente de Posgrados de Varias Universidades, consultor y capacitador en varias instituciones privadas y públicas del país.

ALEX ALVAREZ-ZURITA obtuvo su título Magíster en Tecnología Educativa y Competencias Digitales en la Universidad Internacional de la Rioja (UNIR) España en 2019. Obtuvo el título de Magíster en Educación Superior en la Facultad de Filosofía, Letras de Ciencias de la Educación de la Universidad Central del Ecuador (Ecuador) en 2014 y obtuvo el título de Licenciado en Ciencias de la Educación Mención Informática en la Facultad de Filosofía, Letras de Ciencias de la Educación de la Universidad Central del Ecuador (Ecuador) en 2008.

Actualmente es profesor a medio tiempo de la Facultad de Filosofía, Letras y Ciencias de la Educación de la Universidad Central Ecuador.

DIEGO BRACERO-HUERTAS Bachiller técnico en Administración de Sistemas Informáticos, estudiante de la carrera de Pedagogía de las Ciencias Experimentales Mención Informática en la Facultad de Filosofía, Letras de Ciencias de la Educación de la Universidad Central del Ecuador. 